\title{
Clinical characteristics of critically ill patients with COVID-19 infection: A retrospective single-centre study on 88 patients in the ICU
}

\author{
Jun Feng ${ }^{1}$, Daixing Zhou ${ }^{1}$, Dengxiu Zou ${ }^{1}$, Bangjiang Fang ${ }^{1}$, Wen Zhang ${ }^{1}$, Gang $\mathrm{Yu}^{1}$, \\ Yikuan Feng ${ }^{1}$, Dan Peng ${ }^{1}$, Jifa $\mathrm{Hu}^{1}$, and Junshuai Wang ${ }^{1}$ \\ ${ }^{1}$ Affiliation not available
}

June 16, 2020

\begin{abstract}
Background: Ever since the COVID-19 pneumonia emerged in Wuhan, China, in December 2019, it has rapidly spread worldwide, resulting in high mortality. This study aimed to delineate the clinical characteristics of critically ill patients with COVID-19 infection in the intensive care unit. Methods: In this retrospective study, the clinical characteristics of 88 critically ill patients with COVID-19 infection were collected. Analysis among three subgroups, classified according to the survival time, was performed. Results: The median age was 70.7(62.3-78.25) years of critical ill patients. Hypertension (43.2\%) was the most frequent underlying disease. At the time of admission, compared to other patients, patients of the[?]7 days category showed greater impairment of consciousness, as per the Glasgow Coma Scale (GCS) scores $(11.0 \pm 2.9, \mathrm{P}<0.01)$. The lymphocyte percentages in all patients $(4.1 \pm 0.78)$ were much lower than the normal values. Particularly, acute kidney failure occurred more frequently in patients of the [?]7 days category $(45.2 \%, \mathrm{P}<0.01)$. The time distributions of the mortality rates were 31 deaths in [?]7 days, 33 deaths in $8-14$ days, and7 deaths in $>14$ days $(\mathrm{P}=0.01)$. Conclusion: Critically ill patients with COVID-19 infection showed high risk of mortality. The primary immune response of lymphocytes against the COVID-19 infection was severely destroyed. Higher GCS score predicted greater possibility of survival. However, acute kidney injury may be an indicator of worse outcome.
\end{abstract}

\section{Introduction}

Ever since the new coronavirus (COVID-19) pneumonia emerged in Wuhan, China, in December 2019, it has rapidly spread throughout the world, resulting in high mortality. According to the World Health Organization (WHO) reports, about $80 \%$ of patients with COVID-19 infection have mild symptoms, whereas about $5 \%$ have severe symptoms. The latter group of patients shows more than $50 \%$ mortality. On $11^{\text {th }}$ March 2020, WHO assessed that the COVID-19 infection could be characterised as a pandemic ${ }^{1}$. However, there is insufficient knowledge on the pathophysiological parameters, particularly mortality indicators, to understand the mechanism involved in the COVID-19 infection. Clinical and pathological features of patients with COVID-19 have recently been reported, showing that the COVID-19 infection causes clusters of severe and even fatal pneumonia with a clinical presentation that greatly resembled that of severe acute respiratory syndrome (SARS)-CoV infection. COVID-19 is also associated with admission to the intensive care unit (ICU), and high mortality ${ }^{2,3}$. Several potential drug candidates, including lopinavir/ritonavir, nucleoside analogues, neuraminidase inhibitors, remdesivir, chloroquine, and Chinese traditional medicines (such as Lianhua Qing-wen capsules), have been proposed ${ }^{4,5}$. No vaccine or specific antiviral treatment for the COVID19 infection has been shown to be effective yet; hence, supportive therapy that provides symptomatic relief and protects the multi-organ functions may be beneficial. Identifying or treating high risk patients more promptly is crucial for decreasing the mortality rate. However, lack of clinical data of critically ill patients with COVID-19 promotes this study in Tongji Hospital where was urgently reconstructed and assigned by 
the Chinese government as a hospital designated for severely or critically ill COVID-19 patients.

In this study, we performed a comprehensive analysis and aimed to summarise the severe clinical manifestations of patients with COVID-19 infection. Therefore, this study helped in gaining a better understanding of the risk factors and outcomes of the COVID-19 infection, ultimately helping in guiding the efforts aimed at reducing the fatality rate.

\section{Methods}

Study participants and data collection

This is a retrospective cohort study of 88 critically ill patients, with confirmed COVID-19 infection, admitted in the ICU at Tongji Hospital in Wuhan, China. The study was approved by the Institutional Review Board of Tongji Hospital, Tongji Medical College, Huazhong University of Science and Technology (Wuhan, China).

In January 2020, Tongji Hospital was urgently reconstructed and assigned by the Chinese government as a hospital designated for severely or critically ill COVID-19 patients. All patients were diagnosed according to the Guidelines of diagnosis and management of COVID-19 (6th edition, in Chinese), released by the National Health Commission of China-WHO interim guidance ${ }^{6}$. Critically ill cases were defined based on the following criteria: if respiratory failure occurred and mechanical ventilation was required; if shock occurred; if the infection was complicated with organ failure, requiring monitoring and treatment in the ICU. Information on the dynamic changes in the laboratory variables was collected in the ICU. The study endpoint for this special group of patients was the 28-day mortality in the ICU. According to the survival times, these patients were subdivided into three groups, including the $\leq 7$ days, $8-14$ days, and $>14$ days groups.

Diagnosis and principles of management

Acute respiratory distress syndrome (ARDS), acute kidney injury, sepsis, shock, acute liver injury, and acute cardiac injury were defined according to the interim guidance of WHO for SARS-CoV-2 ${ }^{7}$. All patients received standard intensive medical treatment in the ICU. The standard medications included but were not limited to oxygen support, nutritional support, antiviral therapy, corticosteroid therapy, antibiotics, vasopressors, blood component transfusion, continuous renal replacement therapy (CRRT), and Chinese traditional medicine. Additionally, patients underwent routine blood tests, coagulation and biochemical tests, and chest computed tomography. Supplemental oxygen therapy was given to patients with hypoxaemia immediately. If standard oxygen therapy failed, mechanical ventilation was considered; high flow nasal catheter oxygen or non-invasive ventilation was used. If no improvement was seen within $1 \mathrm{~h}$ of non-invasive mechanical ventilation, invasive mechanical ventilation was provided. Experienced experts recommended extracorporeal membrane pulmonary oxygenation (ECMO) based on their evaluation of the patient's condition. If the patient gave a history of seasonal or local influenza, empirical therapy was considered.

Clinical laboratory measurements

Initial clinical laboratory investigation included a complete blood count, serum biochemical tests (including liver and renal function tests and levels of creatine kinase[CK], lactate dehydrogenase [LDH], and electrolytes), coagulation profile, and levels of serum cytokines, including interleukin (IL)-6, IL-10,and tumour necrosis factor (TNF)- $\alpha$. Arterial blood gas tests were performed within $30 \mathrm{~min}$.

Statistical analysis

All statistical analyses were performed using the Graph Pad Prism (version 7.0 for Windows, Graph Pad Software, La Jolla, California). Continuous variables of the three groups were expressed as mean \pm standard deviation (SD) and compared by one-way ANOVA or non-parametric Kruskal-Wallis tests, and categorical variables of the three groups were expressed as numbers (percentages) and compared by $\chi^{2}$ test or Fisher's exact test. A two- tailed $P$ value $<0.05$ was considered statistically significant.

\section{Results}




\section{Demographic and clinical characteristics of the patients}

The main demographic and clinical characteristics of the 88 patients with a median age of 70.7(62.3-78.3) years are presented in Table 1 . The median age of the patients in the $\leq 7$ days, $8-14$ days, and $>14$ days categories was 74.1 (65.6-80.3), 69.3(60.6-75.3), and 68.3(58.8-74) years, respectively. Most patients were male. All patients developed fever $(37.3 \pm 1.0)$. Nevertheless, the heart and respiratory rates of the patients of the three groups significantly varied; the heart rates were $103+-23.8,91.7+-17.3$, and $91.7+-12.6(P=0.03)$, while the respiratory rates were $27.72+-8.0,27.4+-7.3$, and $23.4+-6.2(P=0.04)$ for the patients of the $\leq 7$ days, 8-14 days, and $>14$ days categories, respectively. The mean arterial pressure (MAP) and pulse oxygen saturation $\left(\mathrm{SpO}_{2}\right)$ of the patients of the three categories were similar. At the time of admission, patients of the 8-14days and >14days categories showed higher Glasgow Coma Scale scores (13.1+-2.6 and 14.3+-1.9, respectively) than those of the [?]7 days category $(11.0+-2.9, P<0.01)$.

The most frequently recorded underlying medical co-morbidities included hypertension (43.2\%), followed by diabetes $(23.9 \%)$, cardiovascular disease (20.5\%), chronic lung diseases (13.6\%), cerebrovascular disease $(3.4 \%)$, chronic kidney disease $(2.3 \%)$, chronic liver disease $(1.1 \%)$, and malignancy (1.1\%). The proportions of co-morbidities and smoking histories of the patients of the three groups were similar. However, acute kidney failure occurred more frequently in the patients of the [?] 7 days category $(45.2 \%)$ than in the patients of the $8-14$ days and $>14$ days categories $(21.2 \%$ and $4.2 \%$, respectively; $P<0.01)$. Among the 88 critically ill patients whose mortality data were reported, 17 survived and 71 died at the end of the study, indicating different rates of mortality for different categories: 31deaths in the $\leq 7$ dayscategory, 33 deaths in the 8-14days category, and 7 deaths in the $>14$ days category. In other words, mortality rates of the patients with three different survival times differed as follows: $57.9 \%$ in $8-14$ days, $35.2 \%$ in $\leq 7$ days, and $29.2 \%$ in $>14$ days $(P=0.01)$. Figures 1 and 2 show the survival curves of the 88 patients and time distributions of their mortality rates.

\section{Laboratory indices}

Laboratory findings provided substantial information on the severity of the disease. White blood cell (WBC) counts $\left(\mathrm{x} 10^{9} / \mathrm{L}\right)$ were elevated in most patients $(11.7+-5.5)$, while the lymphocyte percentages were much lower than the normal value in all patients (4.1+-0.78). Particularly, the lymphocyte percentage in the patients of the [?]7 days category was 5.3+-4.8, which was significantly lower than that in the patients of the $>14$ days category $(9.1+-6.4, P=0.03)$. Red blood cell $(\mathrm{RBC})$ and platelet counts did not differ much from the normal values and among the patients of the three categories. Blood biochemical indices, including alanine aminotransferase (ALT), aspartate aminotransferase (ast), serum creatinine, sodium, potassium, and creatine kinase $(\mathrm{CK})$, also showed no significant deviations from the normal values. In addition, blood urea nitrogen $(\mathrm{BUN})$ and lactate dehydrogenase $(\mathrm{LDH})$ levels in the patients of the $>14$ days category $(8.7+-4.4$ and $434.4+-136.1$, respectively) were significantly lower than those in the patients of the [?]7 days (16.4+10.5and 703.4+-459.2, respectively) and 8-14 days (15.5+-9.9 and 637.8+-331.3, respectively) categories $(P$ $=0.02$ ). Lower levels of CK-MB were found in the patients of the $>14$ days category, compared to that in the patients of the [?]7 days category $(P<0.01)$.

Coagulation function was seriously affected in all patients, showing prothrombin time of $18.7+-16.5$ s. Patients of the $>14$ days category showed shorter prothrombin times(15.1+-1.9) and lower D-dimer levels (4.6+-6.2) than those of the [?]7 days (19.2+-8.9 and 11.6+-8.5, respectively) and 8-14 days (20.9+-24.9 and 11.2+-9.3, respectively) categories $(P<0.01)$. Likewise, patients of the $>14$ days category showed lower C-reactive protein (CRP) (93.9+-54.7) and procalcitonin (PCT) $(0.4+-0.6)$ levels $(P=0.02$ and $P<0.01$, respectively). Levels of serum cytokines, such as IL-6, IL-10, and TNF- $\alpha$, were obviously elevated in all patients, but did not differ among the patients of the three categories. The partial pressure of oxygen $(\mathrm{PaO} 2) /$ fraction of inspirational oxygen (FiO2) index was dramatically decreased in all patients, but did not differ among the patients of the three categories. Baseline laboratory parameters of the patients are shown in Table 2. Constant changes in the routine blood tests are shown in Figure 3. Constant changes in the laboratory parameters and inflammatory biomarkers are shown in Figures 4 and 5, respectively. 
Complications that could be attributed to mortality

Although the patients received aggressive treatment in the ICU, 71 of the 88 patients died within 28 days. Multiple organ dysfunction syndromes (MODS) were prevalent in these patients. Considering one major cause of mortality, we found that 35 deaths $(49.3 \%)$ were attributed to respiratory causes, $23(32.4 \%)$ to cardiovascular causes, $7(9.9 \%)$ to shock, $5(7 \%)$ to kidney injury, and 1(1.4\%) due disseminated intravascular coagulation (DIC), indicating that respiratory failure was the most predominant complication. Figure 6 shows the main causes of mortality.

\section{Discussion}

In this cohort study, we reported the clinical characteristics, risk factors, and clinical outcomes of critically ill patients with COVID-19 infection. Some patients developed MODS after being admitted to the ICU, while some progressed to death. The Chinese Centre for Disease Control reported that most of the confirmed cases were classified as mild or moderate, $13.8 \%$ as severe, and only $4.7 \%$ as critically ill ${ }^{8}$. This was consistent with the WHO report ${ }^{1}$. In recent years, due to the SARS and Middle East respiratory syndrome (MERS) outbreaks, the emergence of the COVID-19 epidemic has caused people to pay attention. The pathogenesis of the highly pathogenic human coronavirus is still not completely understood. Cytokine storm and viral evasion of cellular immune responses are thought to play important roles in disease severity ${ }^{9}$. The severity of lung damage was found to be correlated with extensive pulmonary infiltration of neutrophils and macrophages, and the higher numbers of these cells in the peripheral blood of patients with MERS ${ }^{10}$. Some patients with SARS$\mathrm{CoV}$, MERS-CoV, or COVID-19 infections maybe asymptomatic in the early stages until severe pneumonia, dyspnoea, renal insufficiency, and even death occurs in the later stages ${ }^{11}$. Lymphocytes are essential for the immune responses against viral infections due to their regulatory effect on the positions of leukocytes in the host organs. White blood cell (WBC) counts $\left(\times 10^{9} / \mathrm{L}\right)$ were elevated in most patients $(11.7 \pm 5.5)$, while the lymphocyte percentages in these patients were even lower than data in previous studies ${ }^{12,13}$. Therefore, spectral changes in the activities of lymphocytes may lead to severely maladjusted immune responses.

According to the recent reports on the characteristics of COVID-19 patients who required management in the ICU, advanced age ( $>60$ years), the male sex, and underlying co-morbidities (particularly hypertension) were found to be risk factors for severe COVID-19 infection and death ${ }^{14,15}$. Considering that older age is associated with a decline in immune competence, the results of the present study showed that older age was associated with both MODS and death. Therefore, this relation might be due to the less robust immune response in older patients ${ }^{16}$. The overall fatality rate for the confirmed COVID-19 cases was found to be higher in male patients than in female patients, with an increased risk of death for advanced age in both sexes. The highest fatality rate was observed in patients aged 80 years and above ${ }^{14}$. The 88 critically ill patients with a median age of $70.7(62.3-78.25)$ years present an extremely high mortality rate (80.7\%).

In this study, hypertension (43.2\%) was the most frequent underlying co-morbidity, as seen in older patients. At the time of admission, compared to other patients, patients of the $\leq 7$ days category showed greater impairment of consciousness, as per the GCS scores(11.0 $2.9, P<0.01)$, indicating that higher GCS score predicted greater possibility of survival. Most of the critically ill patients developed leukocytosis and extremely low lymphocyte percentages, but high levels of CRP and PCT, indicating that a large proportion of the critically ill patients might have developed secondary bacterial infection, which could be strongly associated with death, after early COVID-19 infection. Deceased patients, compared with patients of the $>14$ days category, showed persistent and severe lymphopenia, suggesting that a state of lymphocyte immune deficiency was associated with poor prognosis. Although development of respiratory complications was also strongly associated with poor outcome in patients with COVID-19 infection, only $12 \%$ of the patients presented with chronic lung co-morbidities in this study. Considering one major ascribed cause of mortality, we found that respiratory failure was the most common complication (49.3\%), suggesting that the high risk of respiratory failure could not be entirely ascribed to co-existing chronic lung disease. Moreover, cardiovascular event $(32.4 \%)$ was the second most common cause of mortality, indicating that COVID-19 infection could induce acute cardiac injury and injury to other organs. 
To date, no vaccine or specific antiviral treatment for COVID-19 infection has proven to be effective; hence, supportive therapy that eases the symptoms and protects the important organs may be most beneficial. Studies demonstrated that administration of methylprednisolone appeared to reduce the risk of death in patients with $\operatorname{ARDS}^{17,18}$. Consequently, methylprednisolone treatment may be beneficial for those COVID-19 patients who developed ARDS with the progression of the disease. However, Tsai et al. declared that early treatment and high dosing of corticosteroids was associated with significantly increased hospital-related mortality in adult patients with influenza-associated $\mathrm{ARDS}^{19}$. Critically ill patients who have an increased risk of death may develop MODS, including ARDS, sepsis, acute kidney injury, shock, acute cardiac injury, and DIC, which was found to be less frequent $(1.4 \%)$ in the present study. Apart from respiratory failure, acute kidney injury was observed in $25 \%$ of the total patients and $45.2 \%$ of the patients of the [?]7 days category, indicating that acute kidney injury could be a major contributing factor to the risk of fatality of COVID-19 patients, regardless of history of previous kidney disease. Considering the prevalence of MODS in critically ill patients, corticosteroid therapy must be investigated further. In addition, ECMO can be used to maintain oxygenation, improve ventilation, adequately allow the injured lungs to rest, and avoid complications associated with sedation, intubation, and mechanical ventilation. However, ECMO does not provide direct support to the other organs beyond increasing systemic oxygen delivery and mitigating ventilator-induced lung injury ${ }^{20,21}$. Development of respiratory, renal, and cardiac complications, and DIC was also strongly associated with poor outcome of patients with COVID-19 infection ${ }^{22,23}$. Indeed, the survival time of patients who received ECMO was obviously prolonged in our study. Five cases of ECMO were performed, but still one patient died with 14 days.

Taken together, COVID-19 induced injuries of multiple organs, increasing the disease severity and worsening the outcomes ${ }^{24}$. Understanding the clinical features and immune conditions of patients with COVID-19 infection will not only provide a greater insight into the pathogenesis of COVID-19, but will also identify therapeutic targets ${ }^{11}$. Although specific antiviral agents are currently being developed in several countries, the use of antiviral agents alone may not be sufficient to stop multiple organ injuries in patients who present with symptoms in the later stages of the infection. According to the present situation, combined therapies, including Chinese traditional medicines, to protect organ functions and target lymphocyte immunomodulation in order to reduce the inflammation storm, may be used to ameliorate organ injuries and eventually improve mortality ${ }^{25,26}$. However, a limitation of this study is that some laboratory tests were not performed in all patients, and hence, missing data might have led to bias of the statistical analysis. Additionally, the 28-day duration was relatively short.

In conclusion, this is a retrospective single-centre clinical study on a unique population of critically ill patients with COVID-19 infection. The primary immune response of lymphocytes against the COVID-19 infection was severely destroyed. Higher GCS score predicted greater possibility of survival. However, acute kidney injury may be an indicator of worse outcome. These patients presented with fatal clinical manifestations and high risk of mortality, and this helped us in understanding the pathophysiology of COVID-19 infection better. Further studies on the viral factors that drive immune dysregulation and COVID-19 pathogenesis and induce MODS are of great importance for the development of vaccines and specific antiviral agents for the clinical management of the COVID-19 infection.

\section{Acknowledgments}

We thank all the doctors and nurses who are working in the ICU of Tongji hospital and across the nation of China and health service supervisors.

\section{References}

1. Brinkmann V, Zychlinsky A. Neutrophil extracellular traps: is immunity the second function of chromatin? J Cell Biol. 2012;198(5):773-783.

2. Huang C, Wang Y, Li X, et al. Clinical features of patients infected with 2019 novel coronavirus in Wuhan, China. Lancet. 2020;395(10223):497-506. 
3. Yu N, Li W, Kang Q, et al. Clinical features and obstetric and neonatal outcomes of pregnant patients with COVID-19 in Wuhan, China: a retrospective, single-centre, descriptive study. The Lancet Infectious diseases. 2020.

4. Wang M, Cao R, Zhang L, et al. Remdesivir and chloroquine effectively inhibit the recently emerged novel coronavirus (2019-nCoV) in vitro. Cell research.2020;30(3):269-271.

5. Lu H. Drug treatment options for the 2019-new coronavirus (2019-nCoV). Bioscience trends.2020;14(1):6971.

6. Singer M, Deutschman CS, Seymour CW, et al. The Third International Consensus Definitions for Sepsis and Septic Shock (Sepsis-3). JAMA. 2016;315(8):801-810.

7. Scarlatescu E, Tomescu D, Arama SS. Anticoagulant Therapy in Sepsis. The Importance of Timing. $J$ Crit Care Med (Targu Mures). 2017;3(2):63-69.

8. Wu Z, McGoogan JM. Characteristics of and Important Lessons From the Coronavirus Disease 2019 (COVID-19) Outbreak in China: Summary of a Report of 72314 Cases From the Chinese Center for Disease Control and Prevention. Jama. 2020.

9. Channappanavar R, Perlman S. Pathogenic human coronavirus infections: causes and consequences of cytokine storm and immunopathology. Seminars in immunopathology.2017;39(5):529-539.

10. Min CK, Cheon S, Ha NY, et al. Comparative and kinetic analysis of viral shedding and immunological responses in MERS patients representing a broad spectrum of disease severity. Scientific reports. 2016;6:25359.

11. Feng Y, Ling Y, Bai T, et al. COVID-19 with Different Severity: A Multi-center Study of Clinical Features. American journal of respiratory and critical care medicine. 2020.

12. Qin C, Zhou L, Hu Z, et al. Dysregulation of immune response in patients with COVID-19 in Wuhan, China. Clin Infect Dis. 2020.

13. Wang F, Hou H, Luo Y, et al. The laboratory tests and host immunity of COVID-19 patients with different severity of illness. JCI Insight. 2020;5(10).

14. Chen T, Wu D, Chen H, et al. Clinical characteristics of 113 deceased patients with coronavirus disease 2019: retrospective study. Bmj. 2020;368:m1091.

15. Wang D, Hu B, Hu C, et al. Clinical Characteristics of 138 Hospitalized Patients With 2019 Novel Coronavirus-Infected Pneumonia in Wuhan, China. Jama. 2020.

16. Du Y, Tu L, Zhu P, et al. Clinical Features of 85 Fatal Cases of COVID-19 from Wuhan: A Retrospective Observational Study. American journal of respiratory and critical care medicine. 2020.

17. Wu C, Chen X, Cai Y, et al. Risk Factors Associated With Acute Respiratory Distress Syndrome and Death in Patients With Coronavirus Disease 2019 Pneumonia in Wuhan, China.JAMA internal medicine. 2020 .

18. Baek MS, Lee Y, Hong SB, Lim CM, Koh Y, Huh JW. Effect of corticosteroid therapy in the early phase of acute respiratory distress syndrome: a propensity-matched cohort study. The Korean journal of internal medicine. 2020.

19. Tsai MJ, Yang KY, Chan MC, et al. Impact of corticosteroid treatment on clinical outcomes of influenzaassociated ARDS: a nationwide multicenter study. Annals of intensive care. 2020;10(1):26.

20. MacLaren G, Fisher D, Brodie D. Preparing for the Most Critically Ill Patients With COVID-19: The Potential Role of Extracorporeal Membrane Oxygenation. Jama.2020. 
21. Li Y, Cao C, Huang L, et al. "Awake" extracorporeal membrane oxygenation (ECMO) combined with continuous renal replacement therapy (CRRT) for the treatment of severe chemical gas inhalation lung injury. Journal of burn care 85 research : official publication of the American Burn Association. 2020.

22. Chen N, Zhou M, Dong X, et al. Epidemiological and clinical characteristics of 99 cases of 2019 novel coronavirus pneumonia in Wuhan, China: a descriptive study.Lancet. 2020;395(10223):507-513.

23. Lescure FX, Bouadma L, Nguyen D, et al. Clinical and virological data of the first cases of COVID-19 in Europe: a case series. The Lancet Infectious diseases. 2020.

24. Guo YR, Cao QD, Hong ZS, et al. The origin, transmission and clinical therapies on coronavirus disease 2019 (COVID-19) outbreak - an update on the status. Military Medical Research. 2020;7(1):11.

25. Alhazzani W, Moller MH, Arabi YM, et al. Surviving Sepsis Campaign: guidelines on the management of critically ill adults with Coronavirus Disease 2019 (COVID-19). Intensive Care Med. 2020;46(5):854-887.

26. Murthy S, Gomersall CD, Fowler RA. Care for Critically Ill Patients With COVID-19. JAMA. 2020.

Table1. Demographics and clinical characteristics of patients

\begin{tabular}{|c|c|c|c|c|c|}
\hline Parameters & Total $(\mathrm{n}=88)$ & Survival time & Survival time & Survival time & $P$ value \\
\hline & & [?]7 days $(\mathrm{n}=31)$ & $8-14$ days $(\mathrm{n}=33)$ & $>14$ days $(\mathrm{n}=24)$ & \\
\hline Median age (Years) & $70.7(62.3-78.3)$ & $74.1(65.6-80.3)$ & 69.3(60.6-75.3) & $68.3(58.8-74)$ & NS \\
\hline \multicolumn{6}{|l|}{ Sex } \\
\hline Male $^{\mathrm{b}}$ & $60(68.2)$ & $20(64.5)$ & $23(69.7)$ & $17(70.8)$ & NS \\
\hline Female $^{\mathrm{b}}$ & $28(31.8)$ & $11(35.5)$ & $10(30.3)$ & $7(29.2)$ & NS \\
\hline Temperature ()$^{\mathrm{a}}$ & $37.3 \pm 1.0$ & $37.4 \pm 1.2$ & $37.2 \pm 0.9$ & $37.2 \pm 0.9$ & NS \\
\hline Heart rate $(\mathrm{bpm})^{\mathrm{a}}$ & $95.5 \pm 19.4$ & $103 \pm 23.8$ & $91.7 \pm 17.3$ & $91.7 \pm 12.6$ & $0.03^{\mathrm{c}}$ \\
\hline Respiratory rate $(\mathrm{bpm})^{\mathrm{a}}$ & $26.4 \pm 7.4$ & $27.72 \pm 8.0$ & $27.4 \pm 7.3$ & $23.4 \pm 6.2$ & $0.04^{\mathrm{d}}$ \\
\hline $\mathrm{MAP}(\mathrm{mmHg})^{\mathrm{a}}$ & $94.7 \pm 20.6$ & $93.9 \pm 18.7$ & $99.4 \pm 12.4$ & $97.1 \pm 17.1$ & NS \\
\hline $\mathrm{SpO}_{2}{ }^{\mathrm{a}}$ & $87.6 \pm 10.78$ & $88.3 \pm 10.9$ & $87.45 \pm 8.5$ & $86.3 \pm 14.5$ & NS \\
\hline GCS scores ${ }^{\mathrm{a}}$ & $12.7 \pm 2.8$ & $11.0 \pm 2.9$ & $13.1 \pm 2.6$ & $14.3 \pm 1.9$ & $<0.001^{\mathrm{c}}$ \\
\hline Smoking history ${ }^{\mathrm{b}}$ & $9(10.2)$ & $2(6)$ & $4(12.1)$ & $4(16.7)$ & NS \\
\hline \multicolumn{6}{|l|}{ Co-morbidities: } \\
\hline Hypertension $^{\mathrm{b}}$ & $38(43.2)$ & $15(48.4)$ & $13(39.4)$ & $10(41.7)$ & NS \\
\hline Diabetes $^{\mathrm{b}}$ & $21(23.9)$ & $10(32.3)$ & $8(24.2)$ & $1(4.2)$ & NS \\
\hline Cardiovascular disease $^{\mathrm{b}}$ & $18(20.5)$ & $3(9.7)$ & $9(27.3)$ & $6(25)$ & NS \\
\hline Chronic lung diseases $^{\mathrm{b}}$ & $12(13.6)$ & $5(16.1)$ & $5(15.2)$ & $2(8.3)$ & NS \\
\hline Cerebrovascular disease $\mathrm{b}^{\mathrm{b}}$ & $3(3.4)$ & $2(6.5)$ & $0(0)$ & $1(4.2)$ & - \\
\hline Chronic kidney disease $^{\mathrm{b}}$ & $2(2.3)$ & $0(0)$ & $1(3)$ & $1(4.2)$ & - \\
\hline Acute kidney failure ${ }^{\mathrm{b}}$ & $22(25)$ & $14(45.2)$ & $7(21.2)$ & $1(4.2)$ & $<0.01^{\mathrm{e}}$ \\
\hline Chronic liver disease $^{\mathrm{b}}$ & $1(1.1)$ & $1(3.2)$ & $0(0)$ & $0(0)$ & - \\
\hline Malignancy ${ }^{\mathrm{b}}$ & $1(1.1)$ & $0(0)$ & $0(0)$ & $1(4.2)$ & - \\
\hline ECMO & $5(5.7)$ & $0(0)$ & $1(3)$ & $4(16.7)$ & - \\
\hline Count of deaths ${ }^{\mathrm{a}}$ & $71(80.7)$ & $31(35.2)$ & $33(57.9)$ & $7(29.2)$ & $0.01^{\mathrm{e}}$ \\
\hline
\end{tabular}

${ }^{\mathrm{a}}$ Data are expressed as mean $\pm \mathrm{SD}$; and ${ }^{\mathrm{b}}$ data are expressed as numbers (percentages).

MAP, Mean Arterial Pressure; $\mathrm{SpO}_{2}$, Pulse Oxygen Saturation; GCS, Glasgow Coma Scale; ECMO, extracorporeal membrane pulmonary oxygenation.

${ }^{\mathrm{c}} P$ value is obtained by one-way ANOVA, ${ }^{\mathrm{d}} P$ value is obtained by Kruskal-Wallis one-way ANOVA, and ${ }^{\mathrm{e}} P$ value is obtained by Fisher's exact test of contingency tables.

Table 2. Baseline laboratory parameters of the patients at admission 


\begin{tabular}{llllll}
\hline Laboratory tests $($ normal range $)$ & Total $(\mathrm{n}=88)$ & Survival time & Survival time & Survival time & $P$ value \\
\hline & & {$[?] 7$ days } & $8-14$ days & $>14$ days & \\
WBC count $\times 10^{9} / \mathrm{L}(3.5-9.5)$ & $11.7 \pm 5.5$ & $13.2 \pm 6.8$ & $11.27 \pm 6.2$ & $8.5 \pm 4.5$ & $\mathrm{NS}$ \\
RBC count $\times 10^{9} / \mathrm{L}(4.0-5.5)$ & $4.1 \pm 0.78$ & $3.9 \pm 0.73$ & $4.1 \pm 0.7$ & $4.2 \pm 0.9$ & $\mathrm{NS}$ \\
Lymphocyte percentage $\left(20^{\sim} 40\right)$ & $8.5 \pm 5.9$ & $5.3 \pm 4.8$ & $6.9 \pm 5.4$ & $9.1 \pm 6.4$ & 0.03 \\
Platelet count $\times 10^{9} / \mathrm{L}(125-350)$ & $160.7 \pm 89$ & $152.3 \pm 106.3$ & $151.4 \pm 82.5$ & $178.3 \pm 78.6$ & $\mathrm{NS}$ \\
Alanine aminotransferase $(0-40 \mathrm{U} / \mathrm{L})$ & $56.2 \pm 111.9$ & $77.2 \pm 171.8$ & $46.8 \pm 71.8$ & $42.8 \pm 40.3$ & $\mathrm{NS}$ \\
Aspartate aminotransferase $(0-40 \mathrm{U} / \mathrm{L})$ & $80.8 \pm 171$ & $92 \pm 176.2$ & $78 \pm 86.5$ & $65 \pm 62.6$ & $\mathrm{NS}$ \\
Serum creatinine $(53-104 \mu m o l / \mathrm{L})$ & $134.5 \pm 156$ & $139.1 \pm 90.1$ & $144.2 \pm 190.9$ & $114.6 \pm 165.3$ & $\mathrm{NS}$ \\
BUN $(3.1-8.0$ mmol/L) & $12.6 \pm 8.8$ & $16.4 \pm 10.5$ & $15.5 \pm 9.9$ & $8.7 \pm 4.4$ & 0.02 \\
Sodium $(136-145 \mathrm{mmol} / \mathrm{L})$ & $142.3 \pm 10.4$ & $145.3 \pm 11.2$ & $142.1 \pm 6.8$ & $139.6 \pm 13.3$ & $\mathrm{NS}$ \\
Potassium $(3.5-5.1 \mathrm{mmol} / \mathrm{L})$ & $4.57 \pm 2.1$ & $4.6 \pm 0.8$ & $4.4 \pm 0.6$ & $4.7 \pm 0.8$ & $\mathrm{NS}$ \\
Fibrinogen $(24 \mathrm{~g} / \mathrm{L})$ & $4.75 \pm 0.7$ & $4.4 \pm 2.1$ & $4.6 \pm 2.3$ & $5.5 \pm 1.9$ & $\mathrm{NS}$ \\
Prothrombin time $(11.5-14.5 \mathrm{~s})$ & $18.7 \pm 16.5$ & $19.2 \pm 8.9$ & $20.9 \pm 24.9$ & $15.1 \pm 1.9$ & $<0.01$ \\
D-dimer $(<0.5 \mu \mathrm{m} / \mathrm{mL})$ & $4.1 \pm 4.4$ & $11.6 \pm 8.5$ & $11.2 \pm 9.3$ & $4.6 \pm 6.2$ & $<0.01$ \\
C-reactive protein $(<1 \mathrm{mg} / \mathrm{L})$ & $117.5 \pm 71.6$ & $145.2 \pm 61.7$ & $121.3 \pm 95.9$ & $93.9 \pm 54.7$ & 0.02 \\
Procalcitonin $(0.02-0.05 \mathrm{ng} / \mathrm{mL})$ & $1.4 \pm 4.2$ & $1.3 \pm 1.6$ & $2.1 \pm 6.3$ & $0.4 \pm 0.6$ & $<0.01$ \\
Creatine kinase $([?] 190 \mathrm{U} / \mathrm{L})$ & $329.2 \pm 41.4$ & $335.4 \pm 319.8$ & $377.9 \pm 555.4$ & $260.7 \pm 210.6$ & $\mathrm{NS}$ \\
CK-MB $(0-18 \mathrm{U} / \mathrm{L})$ & $18.9 \pm 57.2$ & $14.4 \pm 15.5$ & $32.9 \pm 90$ & $2.2 \pm 2.6$ & $<0.01$ \\
LDH $(135-225 \mathrm{U} / \mathrm{L})$ & $567.8 \pm 297.6$ & $703.4 \pm 459.2$ & $637.8 \pm 331.3$ & $434.4 \pm 136.1$ & 0.02 \\
Interleukin-6 $(<7 \mathrm{pg} / \mathrm{mL})$ & $144.4 \pm 182$ & $523.8 \pm 1212$ & $336 \pm 1054$ & $62 \pm 56.3$ & $\mathrm{NS}$ \\
Interleukin-10 $(<9.1 \mathrm{pg} / \mathrm{mL})$ & $26.7 \pm 42.6$ & $40.6 \pm 64.4$ & $21.2 \pm 22.7$ & $17.59 \pm 9$ & $\mathrm{NS}$ \\
Tumour necrosis factor- $\alpha(<8.1 \mathrm{pg} / \mathrm{mL})$ & $17.3 \pm 14$ & $21.5 \pm 20.5$ & $10.4 \pm 6.9$ & $9.8 \pm 3$ & $\mathrm{NS}$ \\
PaO2/FiO2 $(400-500 \mathrm{mmHg})$ & $120.2 \pm 69.6$ & $110.7 \pm 90.9$ & $113.6 \pm 45.3$ & $157.1 \pm 74.1$ & $\mathrm{NS}$ \\
\hline
\end{tabular}

Data are expressed as mean $\pm \mathrm{SD}$.

WBC, White Blood Cell; RBC, Red Blood Cell; BUN, Blood Urea Nitrogen; CK-MB, Creatine Kinase-MB; LDH, Lactate Dehydrogenase; PaO2, Partial Pressure of Oxygen; FiO2, Fraction of inspiration Oxygen.

$P$ value is obtained by Kruskal-Wallis one-way ANOVA.

Figure 1. Survival curves of the 88 patients

Figure 2. Time distributions of the mortality rates of patients within 28 days

Figure 3. Constant changes in the WBC, RBC and platelet counts and lymphocyte percentages

Figure 4. Constant changes in the blood biochemical indices. ALT, alanine aminotransferase; AST, aspartate aminotransferase; BUN, blood urea nitrogen; PT, prothrombin time; CK-MB, creatine phosphokinase-Mb; $\mathrm{LDH}$, dehydrogenase.

Figure 5. Constant changes in the inflammatory biomarkers. CRP, C-reactive protein; PCT, procalcitonin. Figure 6. The main causes of mortality 

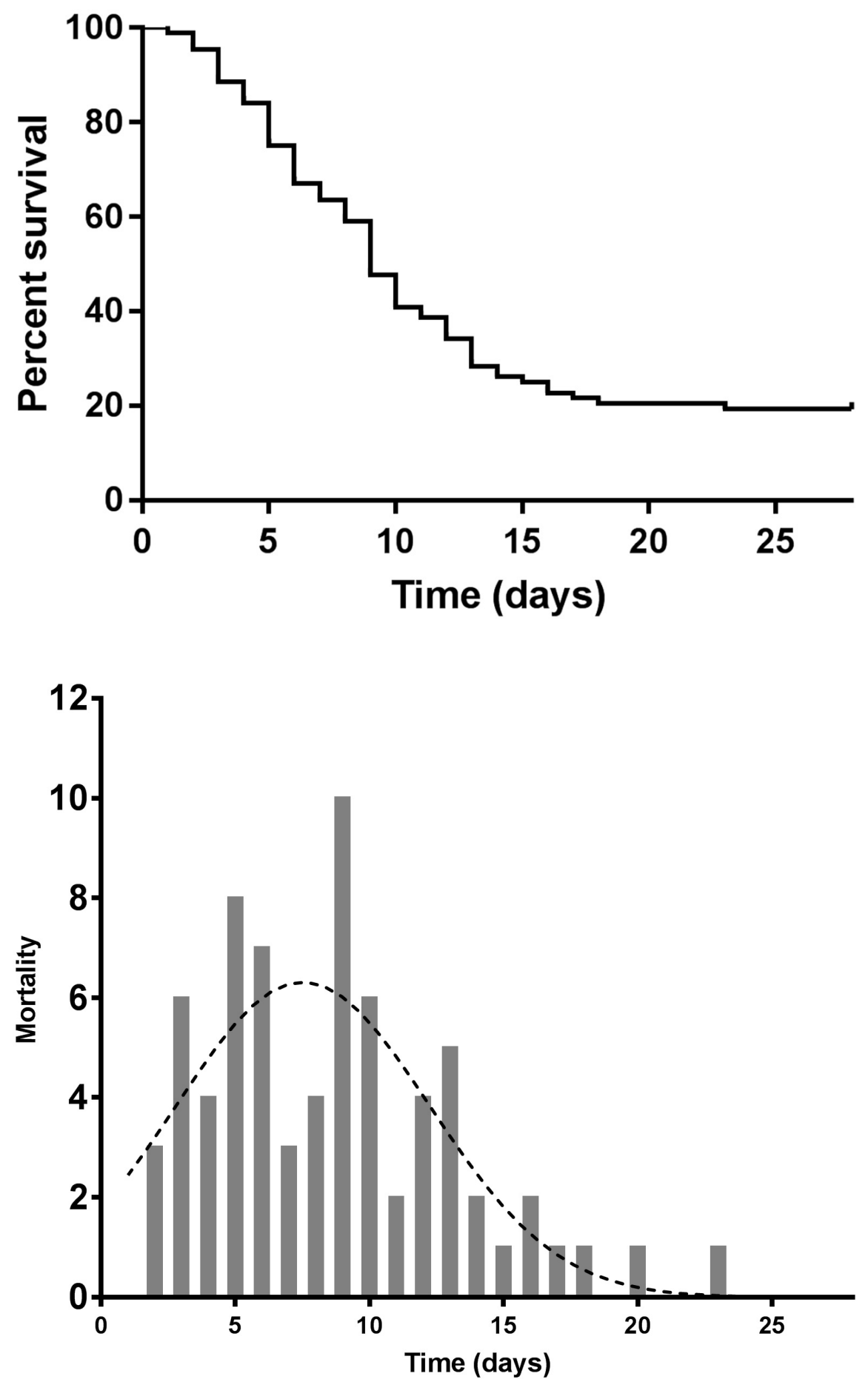
WBC counts

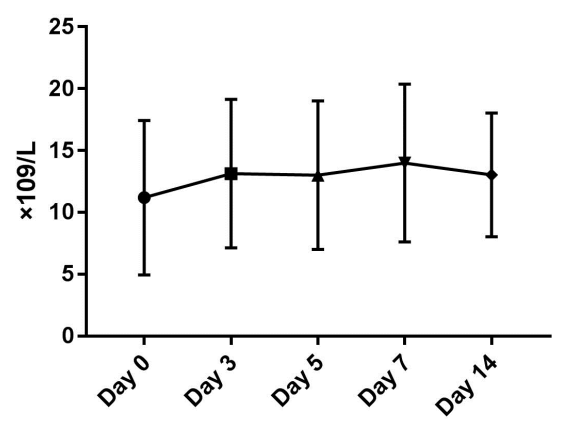

Platelet counts
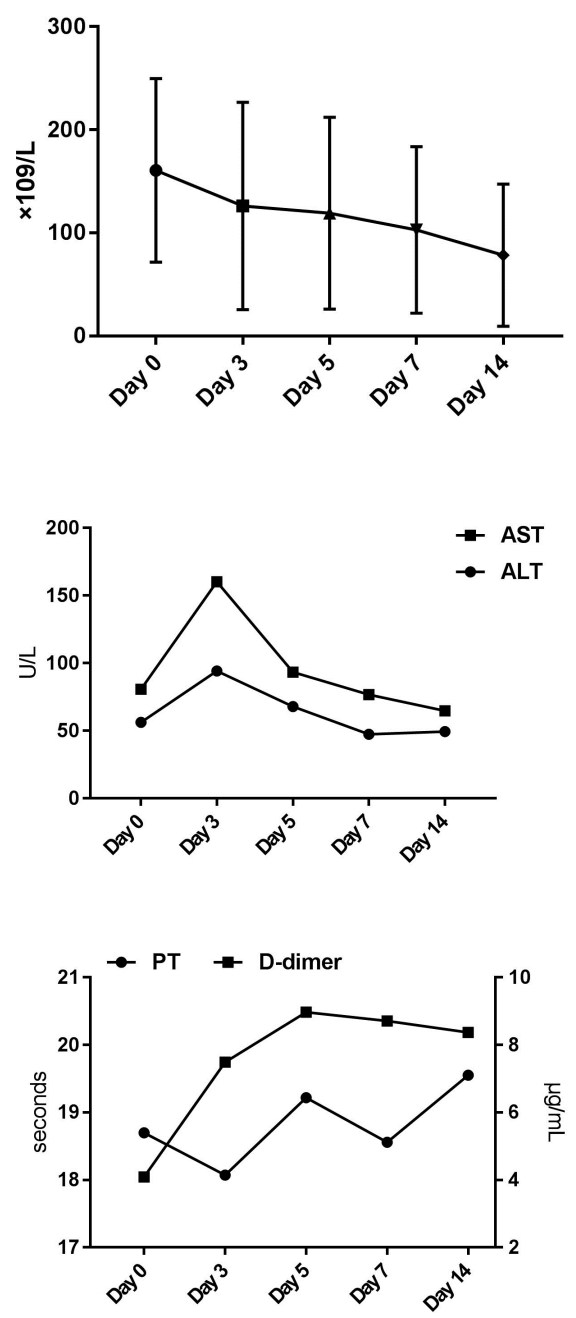

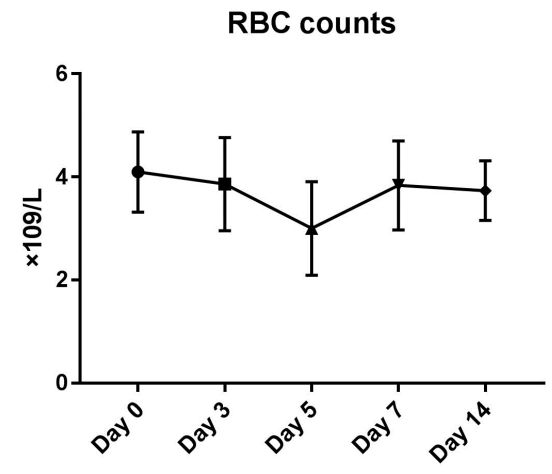

Lymphocyte percentage
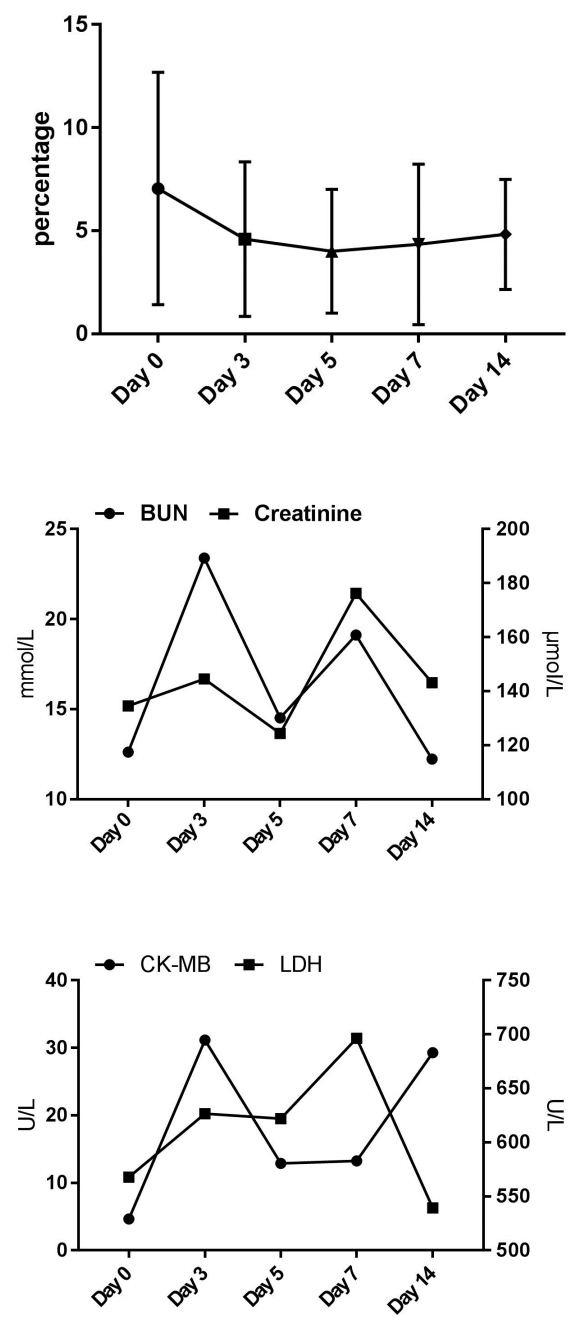

10 

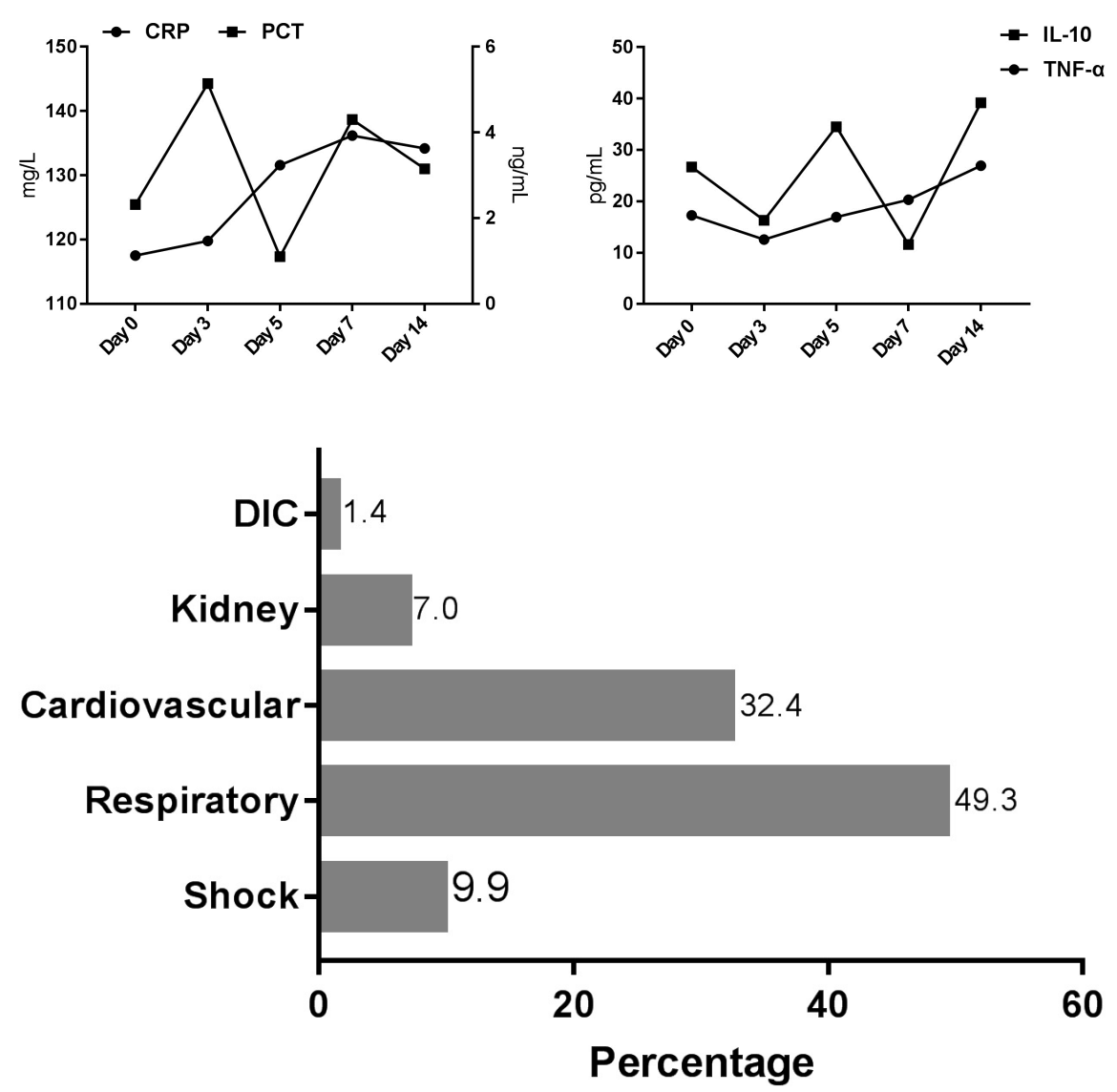